ISSN: $2317-8957$

\title{
RESPOSTA COMPORTAMENTAL DE ECHINASTER (OTHILIA) BRASILIENSIS MÜLLER \& TROSCHEL, 1842, (ECHINODERMATA, ASTEROIDEA) COM BASE EM PREFERÊNCIA ALIMENTAR E OBSERVAÇÃO EM CATIVEIRO
}

\author{
ALESSANDRA ARAUJO DE ALCANTARA ${ }^{1 *}$, FÁBIO MORAES DA COSTA $^{2}$
}

${ }^{1}$ Laboratório de Biologia Marinha. Centro de Pesquisas Biológicas, Universidade Castelo Branco (UCB), Rio de Janeiro, RJ, Brasil. Contato: alessandraalcant@gmail.com

${ }^{2}$ Universidade Castelo Branco, Escola da Saúde e Meio Ambiente. Rio de Janeiro, RJ, Brasil. Contato: fmcchemist@gmail.com

\section{RESUMO}

A Echinaster brasiliensis é uma espécie de estrela-do-mar, da família Echinasteridae, que em sua dieta estão inseridos animais vivos e mortos, especialmente caramujos, bivalves,crustáceos, poliquetas, outros equinodermos e peixes. São descritas como espongívoras, mas não consomem somente estes organismos, consumindo também, artêmias e anêmonas. O presente estudo avalia a resposta comportamental de E. brasiliensis baseada na preferência alimentar. Indivíduos adultos coletados in loco, foram transportados para o Laboratório de Biologia Marinha, Centro de Pesquisas Biológicas, Universidade Castelo Branco, RJ, para observação de comportamento alimentar. Os resultados indicaram aceitação por preferência completa do camarão.

Palavras-chave: Alimentação, Comportamento, Echinasteridae, Estrela-do-mar.

\begin{abstract}
Echinaster brasiliensis is a species of starfish from the Echinasteridae family, which contains live and dead animals, especially snails, bivalves, crustaceans, polychaetes, other echinoderms and fish. They are described as spongivorous, but do not consume only these organisms, consuming also, artemia and anemones. The present study evaluates the behavioral response of E. brasiliensis based on food preference. Adult subjects collected in loco were transported to the Marine Biology Laboratory, Biological Research Center, University Castelo Branco, RJ, to observe feeding behavior. The results indicated acceptance by complete shrimp preference.
\end{abstract}

Keywords: Feeding, Behavior, Echinasteridae, Starfish.

\section{INTRODUÇÃO}

A Classe Asteroidea representa o segundo maior grupo do Filo Echinodermata com uma estimativa de cerca de 1900 espécies vivas (Mah e Blake, 2012). Gondim et al., (2014), afirma que no Brasil, atualmente são conhecidas por volta de 80 espécies, sendo Echinaster brasiliensis a espécie de estrela-do-mar mais conhecida.

São animais bentônicos e ocupa diversos tipos de substrato, sendo predominantemente marinho, adaptando-se para se fixarem à substratos rochosos, lodosos, arenosos, e/ou em madeira submersa (LACOUTH, 2015). 
Gondim et al., (2015), descreve que estrelas-domar do gênero Echinaster são comuns em recifes ao longo de toda a costa brasileira, sendo também raramente encontrado em ambientes estuarinos.

Sua alimentação é basicamente constituída por animais vivos e mortos, onde caramujos, bivalves, crustáceos e poliquetas, além de outros equinodermos e, até mesmo, peixes quando a presa vem descansar sobre a superfície aboral (RUPPERT et al., 2005).

De acordo com Gondim (2008), desempenham importantes papéis ecológicos nas comunidades marinhas, pois muitos são capazes de controlar as densidades populacionais de determinadas espécies, e atuam na reciclagem de nutrientes e nos processos bioerosivos.

Com relação a sua morfologia, apresentam um disco central por onde saem os braços, adquirindo um formato de estrela propriamente dito, e os números de braços podem ser bastante variável, indo de 5 até 50 (RIBEIRO-COSTA e ROCHA, 2006).

De acordo com Barker e Nichols (1983), a estrela-do-mar juvenil alimenta-se de forma carnívora na conclusão da metamorfose. A boca em E. brasiliensis está localizada no centro do lado inferior do disco, que se chama "superfície oral" (Figura 1), e que está em contato com o substrato (RUPPERT et al., 2005).

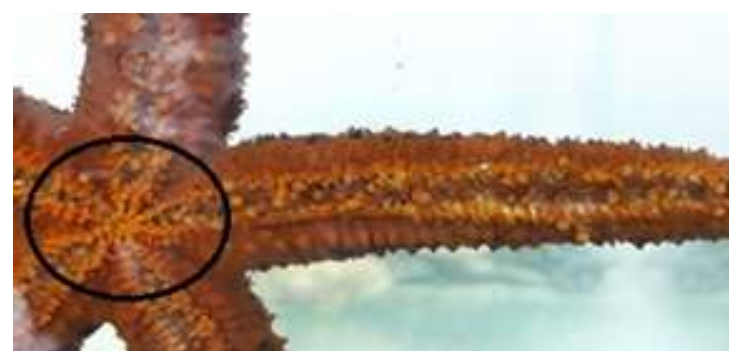

Figura 1. Superfície oral sinalizado em preto.

A boca se abre para um curto esôfago que leva um grande estômago (Figura 2), no qual ocupa a maior parte do disco, liberando enzimas digestivas e envolvendo a presa, que é parcialmente digerida podendo ser levada para dentro do estômago; quando a alimentação termina, os músculos estomacais se contraem, retraindo o estômago para dentro do disco (RUPPERT et al., 2005).

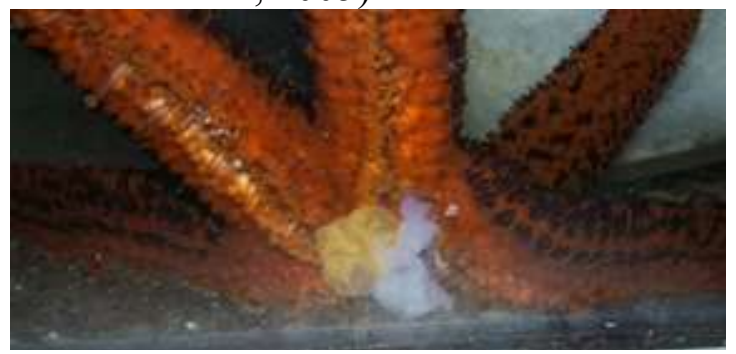

Figura 2. Exemplar de E. brasiliensis. Sinalizando o estômago evertido para alimentação.

Espécies da família Echinasteridae são descritas como espongívoras, mas não consomem somente estes organismos, consumindo também, artêmias e anêmonas. (QUEIROZ, 2012).

$\mathrm{O}$ aparato digestivo em Echinaster pode funcionar efetivamente na coleta de nutrientes de uma variedade de fontes, consumindo nutrientes dissolvidos liberados por meio de atividade digestiva externa modesta ou obtidos de outras fontes naturais, como animais em decomposição, detritos ou água do mar rica em orgânicos (FERGUSON, 1969).

De acordo com Ruppert et al., (2005), além de possuírem uma dieta de ampla variedade de captura de presas, podem apresentar uma preferência alimentar, dependendo da disponibilidade ao ambiente. Porém, de acordo com Pratchett (2001), identificar a presa preferida no campo é difícil porque as preferências alimentares são confundidas por diferenças no tamanho relativo, abundância e acessibilidade.

$\mathrm{O}$ presente estudo tem como objetivo avaliar a resposta comportamental de E. brasiliensis baseada na preferência alimentar.

\section{METODOLOGIA}

Nobre e Campos-Creasey (2010), estimam-se que as E. brasiliensis necessitariam de aproximadamente 14 anos para atingir um tamanho de $\mathrm{R}=50 \mathrm{~mm}$ (distância entre a boca e a extremidade do maior braço - Raio maior) se submetidas a condições de aporte nutricional reduzido e salinidade controle (33,5 psu) e mantidas em aquários com filtro biológico de cascalho em condições laboratoriais. 
Foi montada uma metodologia aplicada à coleta autorizada pelo IBAMA sob o ${ }^{\circ}$ 3611513, na Praia da Viola, na Ilha de Itacuruçá, RJ. O mergulho na modalidade livre (apneia) foi utilizado para a coleta dos indivíduos. Totalizando-se 23 indivíduos adultos coletados in loco, acomodados em recipientes de polietileno hermeticamente fechados com água do mar para manter a integridade física do animal, e em seguida transportados até o Laboratório de Biologia Marinha (LabMar), Centro de Pesquisas Biológicas, Universidade Castelo Branco, RJ. Os indivíduos foram dispostos em 8 aquários com volume de 10L cada para observação, com iluminação por fotoperíodo entre 4 a 6 horas ao dia, com lâmpadas fluorescentes de $20 \mathrm{~W}$.

As etapas da alimentação consistiram após um período de $48 \mathrm{~h}$ à coleta dos indivíduos. Foram ofertados seis tipos de alimentos, referentes à dieta de E. brasiliensis de forma alternada a cada dois dias: misidáceos, esponja-domar, alga Ulva, anêmona-do-mar, artêmias, e camarão cinza fresco descascado.

Dos 23 indivíduos, 22 foram monitorados em um período de dois meses, de junho a agosto de 2017, sendo devolvidos ao ambiente natural ao final dos ensaios. Apenas 1 indivíduo condicionado foi mantido durante seis meses em cativeiro, de junho a dezembro de 2017 , para análise comportamental e condicionamento alimentar de acordo com a sua preferência, sendo devolvido ao ambiente natural ao final dos ensaios.

\section{RESULTADOS E DISCUSSÃO}

Os resultados apontaram para preferência alimentar do camarão cinza descascado, e em seguida para os misidáceos e artêmias respectivamente. Os resultados indicaram aceitação completa do camarão.

A Figura 3 apresenta o percentual de misidáceos ofertados/consumidos por indivíduo.

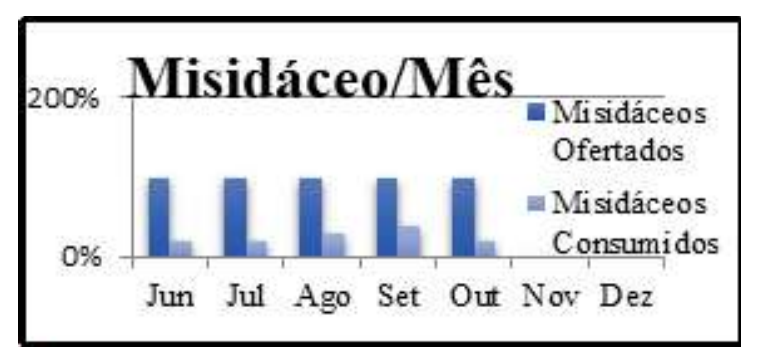

Figura 3- Gráfico representativo da alimentação com misidáceos por mês.

Os resultados na Figura 4 apresenta o percentual de artêmia ofertada/consumida por E. brasiliensis. Nos meses de junho e julho, o percentual inicial do alimento consumido foi de $20 \%$, enquanto no mês de agosto $30 \%$, com $40 \%$ no mês de setembro e voltando com $20 \%$ de animais consumidos no mês de outubro. Nos meses de novembro e dezembro não foi ofertada artêmias na dieta de E. brasiliensis por ajustes do consumo do alimento para que não houvessem sobras.

O consumo de misidáceos e artêmias se mantiveram na mesma proporção durante o experimento.

A Figura 4 apresenta o percentual de artêmia ofertada/consumida por indivíduo.

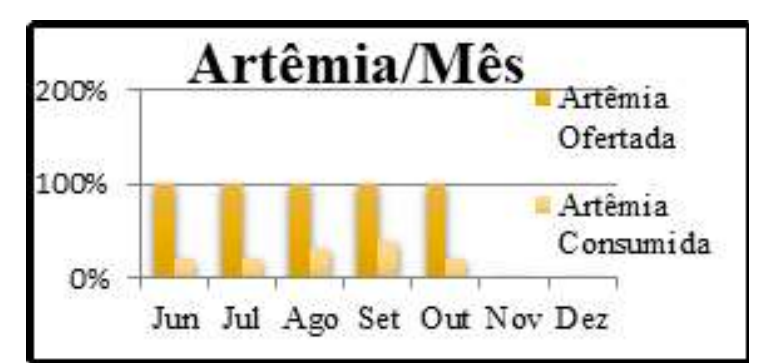

Figura 4 - Gráfico representativo da alimentação com artêmias por mês.

Entretanto, para as doses de camarão cinza oferecido da dieta de Echinaster brasiliensis tiveram proporções diferentes dos demais alimentos ofertados, tendo um bom condicionamento alimentar. Como mostra a Figura 5 nos meses de junho, julho, agosto, setembro e outubro, o consumo de camarão cinza descascado foi de $100 \%$ em relação ao alimento ofertado. Tendo em vista que, todo alimento era consumido por completo, teve um aumento da dose diária chegando a $150 \%$ durante os meses de 
novembro e dezembro após a suspensão dos misidáceos e artêmias, por este modo, para que todo alimento oferecido fosse consumido de maneira que não houvessem sobras e que desta forma não alterasse a composição físico-química da água.

A Figura 5 apresenta o percentual de camarão ofertado/consumido por indivíduo.

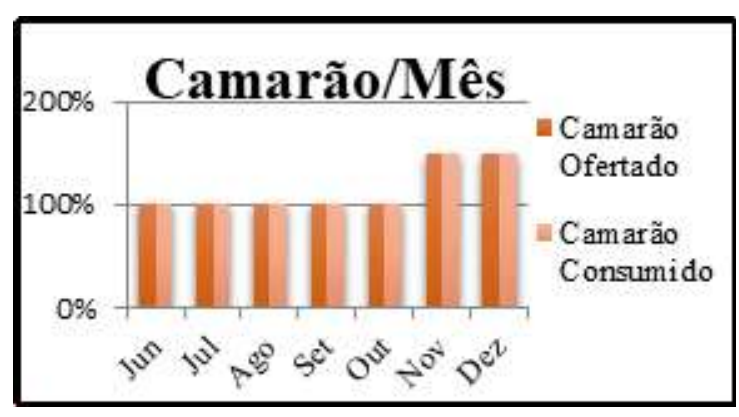

Figura 5 - Gráfico representativo da alimentação com camarão cinza fresco descascado por mês.

\section{CONCLUSÃO}

Conclui-se que E. brasiliensis mantida em cativeiro teve sua dieta restritamente baseada nas condições alimentares das quais apresentaram melhor resposta comportamental, baseada desta maneira, primeiramente na preferência alimentar de camarão cinza, seguidos de misidáceos e artêmias. Considerando que a preferência alimentar para os crustáceos se dá ao maior aporte nutricional para as condições comportamentais em cativeiro para Echinaster brasiliensis, uma vez que não há estudos comparativos de preferência alimentar em cativeiro em literatura. A esponja-do-mar, a alga Ulva e a anêmona-do-mar foram excluídos da dieta, por rejeição completa na alimentação.

\section{REFERÊNCIAS BIBLIOGRÁFICAS}

BARKER, M. F.; NICHOLS, D. Reproduction, recruitment and juvenile ecology of the starfish, Asterias rubens and Marthasterias glacialis. Journal of the Marine Biological Association of the United Kingdom, v. 63, n. 4, p. 745-765, 1983.
FERGUSON, J. C. Feeding activity in Echinaster and its induction with dissolved nutrients. The Biological Bulletin, v. 136, n. 3, p. 374384, 1969.

GONDIM, A. I.; LACOUTH, P.; ALONSO, C.; MANSO, C. L. C. Echinodermata da Praia de Cabo Branco, João Pessoa, Paraíba, Brasil. Biota Neotrop., vol. 8, no. 2, Abr./Jun. 2008.

GONDIM, A. I.; CHRISTOFFERSEN, M. L.; DIAS, T. L. P. Taxonomic guide and historical review of starfishes in northerastern Brazil (Echinodermata, Asteroidea). ZooKeys, v.449, 1-56, 2014.

GONDIM, A. I.; DIAS, T. L. P; CHRISTOFFERSEN, M. L. Hypersaline mangroves harbor a high density of Brazilian endangered sea stars (Echinaster spp.). Marine Biodiversity, v. 45, n. 4, p. 629-630, 2015.

LACOUTH, P. S. Efeitos das mudanças climáticas na regulação de biomarcadores em Echinaster brasiliensis (Echinodermata: Asteroidea) 2015. Tese de Doutorado em Ciênicas na Área de Fisiologia Geral ao Instituto de Biociências da Universidade de São Paulo. São Paulo, 2015.

Mah CL, Blake DB (2012) Global diversity and phylogeny of the Asteroidea (Echinodermata).

PLoS ONE 7: e35644. doi: 10.1371/journal. pone. 0035644

NOBRE, C. C.; CAMPOS-CREASEY, L. S. Efeito da salinidade no desenvolvimento larval de Echinaster echinophorus (Lamarck 1816) (Echinodermata: Asteroidea). Brazilian Journal of Aquatic Science and Technology, v. 4, n. 1, p. 61-68, 2010.

PRATCHETT, M. S. Influence of coral symbionts on feeding preferences of crown-of-thorns starfish Acanthaster planci in the western Pacific. Marine Ecology Progress Series, v. 214, p. 111-119, 2001. 
QUEIROZ, C. H. S. Estratégias e fisiologia do consumo e digestão de esponjas (Porífera) por Echinaster brasiliensis (Echinodermata: Asteroidea) 2012. Tese de Mestrado em Ciências na Área de Fisiologia Geral ao Instituto de Biociências da Universidade de São Paulo. São Paulo, 2012.

RIBEIRO-COSTA, C. S.; ROCHA, M. R. Invertebrados. $2^{\circ}$ Edição. Ribeirão Preto, SP., Brasil. Editora Holos, 2006. 271p.

RUPPERT, E. E.; FOX, R. S.; BARNES, R. D. Zoologia dos Invertebrados. $7^{\circ}$ EDIÇÃO. São Paulo, SP. Editora Roca Ltda., 2005. 1145p.

SLIVAK, N. N. FAUNA ECHINODERMATA DE SANTA CATARINA, BRASIL: Levantamento faunístico e características populacionais de Echinaster (Othilia) brasiliensis Müller \& Troschel, 1842. 2013. Dissertação de mestrado apresentada ao Programa de Pós-Graduação em Biologia Animal da Universidade Federal do Rio Grande do Sul, como parte dos requisitos necessários à obtenção do título de Mestre em Biologia Animal. Universidade Federal do Rio Grande do Sul, Porto Alegre. Rio Grande do Sul, Brasil, 2013. 